\title{
Shear bond strengths of composite resin and giomer to mineral trioxide aggregate at different time intervals
}

\author{
Amir-Ahmad Ajami ${ }^{1}$, Mahmoud Bahari ${ }^{1,2}$, Arezoo Hassanpour-Kashani ${ }^{3}$, Mehdi Abed-Kahnamoui ${ }^{4}$, Ayda \\ Savadi-Oskoee ${ }^{5}$, Farhad Azadi-Oskoee ${ }^{5}$
}

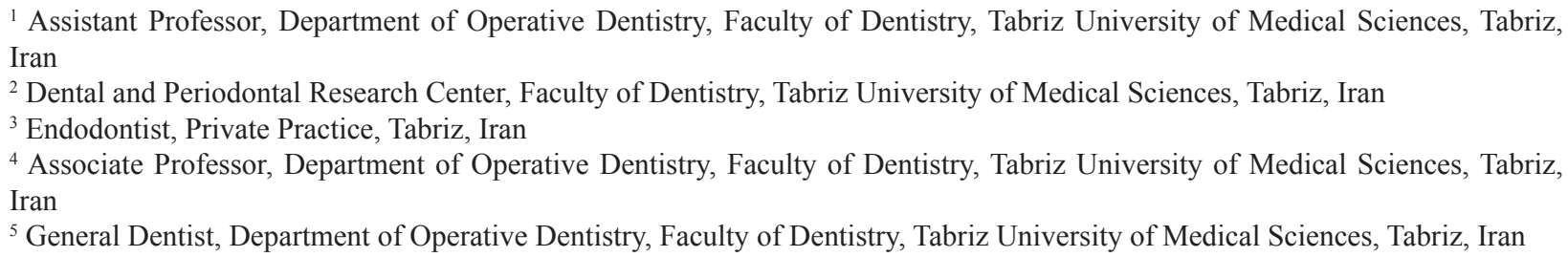

Correspondence:

Dental and Periodontal Research Center and Department of Operative Dentistry

Faculty of Dentistry, Tabriz University of Medical Sciences

Tabriz, Iran

mahmoudbahari@ymail.com

Ajami AA, Bahari M, Hassanpour-Kashani A, Abed-Kahnamoui M, Savadi-Oskoee A, Azadi-Oskoee F. Shear bond strengths of composite resin and giomer to mineral trioxide aggregate at different time intervals. J Clin Exp Dent. 2017;9(7):e906-11.

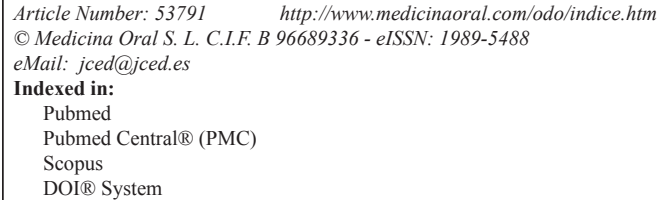

\begin{abstract}
Background: The efficacy of the bond between the restorative materials and the pulp capping materials has an important role in the success of vital pulp therapy. Therefore, the aim of this study was to evaluate the shear bond strength of composite resin and giomer to MTA at different time intervals after mixing of MTA.

Material and Methods: Ninety cylindrical MTA samples were prepared and assigned to two groups $(n=45)$ based on the restorative materials used (composite resin or giomer). Each group was subdivided into 3 subgroups ( $\mathrm{n}=15$ ) based on the evaluation intervals (immediately, 2.45 hours and 3 days after mixing MTA). After the bonding procedures, the shear bond strengths of the samples were measured in MPa at a strain rate of $0.5 \mathrm{~mm} / \mathrm{min}$. Data were analyzed with repeated-measures ANOVA, post hoc tests and t-test $(P<0.05)$.

Results: Bond strength of composite resin was minimum at baseline but it increased significantly 2.45 hours after mixing MTA $(P=0.002)$, with no significant changes in bond strength up to three days $(P=0.08)$. Bond strength of giomer did not exhibit any significant changes from baseline to 2.45 hours after mixing MTA $(\mathrm{P}=078)$; however, at 3 days it reached a minimum $(P=0.000)$. In addition, the means of bond strength of composite resin 2.45 hours and 3 days after mixing were significantly higher than those of giomer ( $P=0.001$ and $P=0.000$, respectively).

Conclusions: Bond strengths of composite resin 2.45 hours and also 3 days after mixing were significantly higher than those of giomer. In addition, the shear bond strength of giomer decreased over time; however, the shear bond strength of composite resin increased.
\end{abstract}

Key words: Composite resin, Giomer, Shear bond strength, Vital pulp therapy. 


\section{Introduction}

In recent years, vital pulp therapy has attracted special attention in dentistry, especially in endodontics (1). The aim of vital pulp therapy is to preserve the health and vitality of the dental pulp after traumatic injuries and exposure of the pulp due to caries (2). Historically, this treatment modality is carried out with the use of calcium hydroxide; however, due to its unpredictable results, it has not been widely accepted and adopted (3).

Introduction of new dental materials, which is supported by scientific evidence, has resulted in an increase in the cases of vital pulp therapy in recent years $(1,4)$. In this context, mineral trioxide aggregate (MTA) has attracted special attention in vital pulp therapy, which is due to its proper biologic properties and favorable clinical and histological outcomes (5). MTA is superior to calcium hydroxide in relation to the induction of dentinogenesis in human dental pulp tissue (6). In addition, the pulp heals faster with the use of MTA compared to calcium hydroxide (7). In direct pulp cap (DPC) procedures, MTA has exhibited better efficacy compared to calcium hydroxide (8).

An important consideration is the fact that after a DPC procedure the restoration area should be completely sealed with an appropriate restorative material to prevent subsequent bacterial contamination $(9,10)$. However, the majority of researchers who have suggested the use of MTA for vital pulp therapy purposes have recommended no restorative material and adhesive system as appropriate for permanent restoration of the cavity, nor have they suggested any time interval as appropriate for the final restoration after mixing and placing it in the pulp cap area (11).

In one of the few relevant studies, Tunch et al. showed that the etch-and-rinse adhesive systems exhibited higher shear bond strength between MTA and composite resins compared to the self-etch systems (12). On the contrary, Neelakantan et al. concluded that the bond strength of composite resin samples with a single-step self-etch bonding protocol immediately after mixing MTA was significantly higher than that achieved with etch-andrinse and two-step self-etch systems at 45-minute and 24-hour intervals after mixing (11).

During the past decade, a new group of restorative materials, referred to as giomers, has been introduced. Giomers are similar to conventional methacrylate-based composite resins; however, they contain inorganic fillers, measuring 0.01 to $0.5 \mu \mathrm{m}$, of pre-reacted glass-ionomer instead of pure glass or quartz fillers $(13,14)$. Giomers are used in a manner similar to conventional composite resins, with the application of an adhesive system. In addition, they exhibit favorable esthetic appearance, easy polishing, strength, and release and recharging of fluoride. The clinical success of giomer restorations has been shown in various studies $(13,15)$. Considering a lack of sufficient data in relation to comparison of the bond strength of giomers and composite resins to MTA, the present study was designed to evaluate and compare the shear bond strength of composite resin and giomer to MTA at different time intervals after mixing MTA, i.e. immediately, 2 hours and 45 minutes, and 3 days after mixing.

\section{Material and Methods}

Sample size calculation

Based on a study by Neelakantan et al., the sample size was calculated at 90 (45 in each group) by considering $\mu 2=38.5, \mu 1=38.4, \mathrm{~S} 1=1.3, \mathrm{~S} 2=1.91, \alpha=0.05$, study power $=80 \%$ and $\mathrm{d}=0.75(11)$.

-Study Design

After the study protocol was approved by the Ethics Committee of the University, first 90 cylindrical acrylic resin samples, measuring $1 \mathrm{~cm}$ in diameter and $25 \mathrm{~cm}$ in height, were prepared. In order to create a completely smooth and horizontal surface, the cylinders were meticulously trimmed in a trimmer. Then cavities were created at the center of the cylinders, measuring $4 \mathrm{~mm}$ in diameter and $2 \mathrm{~mm}$ in height.

The MTA powder was mixed with distilled water based on manufacturer's instructions in a Dappen dish to a thick creamy consistency. Then the paste was placed in the cavities in the acrylic resin molds using a carrier and packed with a condenser, followed by smoothening its surface with a spatula. To create identical surface roughness in all the MTA samples, the surfaces were polished with 600 -grit abrasive paper before the bonding procedures.

Then the samples were randomly assigned to two groups based on the restorative material used: composite resin or giomer $(n=45)$. Then the samples in each group were subdivided into 3 subgroups $(\mathrm{n}=15)$ based on the setting procedure duration: immediately, 2.45 hours and 3 days as follows:

Subgroup 1: Composite resin, immediately

Subgroup 2: Composite resin, 2.45 hours

Subgroup 3: Composite resin, 3 days

Subgroup 4: Giomer, immediately

Subgroup 5: Giomer, 2.45 hours

Subgroup 6: Giomer, 3 days

In MTA samples of subgroups 1 and 4, the bonding procedures were carried out immediately after placing MTA in the cavities of acrylic resin molds. All the other samples were placed vertically within a plastic container and $1 \mathrm{~cm}$ of water was poured in the container. After placing the lid of the container tightly, the samples in subgroups 2 and 5 and in subgroups 3 and 6 were incubated at $37^{\circ} \mathrm{C}$ for 2.45 hours and 3 days, respectively.

In subgroups 1, 2 and 3, in order to bond composite resin samples to MTA surfaces, the surfaces of MTA samples were etched with $35 \%$ phosphoric acid gel (Scotchbond 
Etchant, 3M ESPE, St Paul, MN, USA) for 15 seconds, rinsed with water for 30 seconds and then dried with oiland moister-free air spray.

In the next stage, a clean microbrush (Microbrush Co, Greyton, WI, USA) was used to apply Adper Single Bond IITM one-bottle adhesive (3M ESPE, St Paul, MN, USA) to the prepared surfaces of the samples. The adhesive was applied in two layers based on manufacturers' instructions and after application of each layer a mild air stream was applied on it for 2-5 seconds in order to vaporize the solvent. Then the adhesive layer was light-cured for 10 seconds using a Demetron A2 light-curing unit (KEEP CORPORAION 3225 Deming Way Suite 190 Middleton WI) at a light intensity of $1000 \mathrm{~mW} / \mathrm{cm}^{2}$.

In subgroups 4, 5 and 6, in order to bond giomer to MTA surfaces, the self-etch Beautibond bonding agent (Shofu Inc., Kyoto 605-0983, Japan) provided by the giomer manufacturer was used. An adequate amount of the bonding agent was applied on the prepared MTA surfaces with the use of a clean microbrush. After 10 seconds, a 3-second mild air stream and then a strong current of air were applied to learn a third homogeneous adhesive layer. The Demetron A2 light-curing unit was used to light-cure the adhesive layer for 5 seconds at a light intensity of $1000 \mathrm{~mW} / \mathrm{cm}^{2}$.

To achieve identical surfaces of composite resin and giomer in all the samples, transparent molds, measuring $3 \mathrm{~mm}$ in diameter and height, were used. To this end the transparent mold was placed on the surface of prepared MTA samples and in samples in group 1, Valux Plus composite resin (3M ESPE, St Paul, MN, USA) and in samples in group 2, giomer (Shofu Inc., Kyoto 605-0983, Japan) were placed within the mold with the use of a condenser, followed by light-curing for $20 \mathrm{se}-$ conds with the use of Demetron A2 light-curing unit at a light intensity of $1000 \mathrm{~mW} / \mathrm{cm} 2$ from the occlusal aspect; finally, the whole mass was light-cured again for 40 seconds. Then the samples were incubated at $37^{\circ} \mathrm{C}$ and $100 \%$ relative humidity for 24 hours (11).

-Shear bond strength testing

In the final stage, the shear bond strength of the samples were determined in MPa in a universal testing machine
(Hounsfield Test Equipment, Model H5KS, Surrey, UK) at a strain rate of $0.5 \mathrm{~mm} / \mathrm{min}$.

-Fracture pattern determinations

Failure modes were determined under a stereomicroscope at $\times 10$ and based on observations were divided into two adhesive or cohesive failure modes (Fig. 1).

-Statistical analysis of data

Data were analyzed with repeated-measures ANOVA, LSD tests and t-test, using SPSS 20. Statistical significance was set at $P<0.05$.

\section{Results}

Table 1 presents the means and standard deviations of shear bond strength values of composite resin and giomer to MTA at different time intervals and the failure modes in the study groups.

Kolmogorov-Smirnov test showed normal distribution of data $(P=0.93)$. In repeated measurements, considering the results of Mauchly's sphericity test (the hypothesis of covariance homogeneity) $(P=0.58)$, Greenhouse-Geisser test was used to evaluate the shear bond strength over time; the results showed that during the 3-day period the shear bond strength of composite resin $(P=0.001)$ and giomer $(P=0.000)$ to MTA changed significantly. Therefore, LSD tests were used for two-by-two comparisons of the time intervals in relation to shear bond strength values.

Evaluation of the shear bond strength of composite resin to MTA over time with LSD tests showed that:

-The shear bond strength immediately after mixing MTA was significantly less than that 2.45 hours $(P=0.002)$ and 3 days $(P=0.03)$ after mixing.

-The shear bond strengths 2.45 hours and 3 days after mixing were not significantly different $(P=0.08)$ (Fig. 1).

In addition, evaluation of the bond strength of giomer to MTA over time with LSD tests showed that:

-The shear bond strength immediately and 2.45 hours after mixing were not significantly different $(P=0.78)$.

- The shear bond strength 3 days after mixing was significantly less than that immediately $(P=0.000)$ and 2.45 hours after mixing $(P=0.000)$ (Fig. 1$)$.

Table 1: The means \pm standard deviations and failure modes of shear bond strength tests.

\begin{tabular}{|c|c|c|c|c|c|c|c|c|c|}
\hline \multirow[t]{3}{*}{ Material } & \multicolumn{3}{|c|}{ Shear Bond Strength (Mean \pm SD) } & \multicolumn{6}{|c|}{ Failure modes (\%) } \\
\hline & \multirow[t]{2}{*}{ Immediately } & \multirow[t]{2}{*}{2.45 hours } & \multirow[t]{2}{*}{3 days } & \multicolumn{2}{|c|}{ Tmmediately } & \multicolumn{2}{|c|}{2.45 hours } & \multicolumn{2}{|c|}{3 days } \\
\hline & & & & Coh & Adh & Coh & Adh & Coh & Adh \\
\hline Composite & $2.76 \pm 1.07^{* a}$ & $4.48 \pm 0.81^{* \mathrm{~b}}$ & $3.70 \pm 0.91^{* b}$ & 100 & - & 100 & - & 100 & - \\
\hline Giomer & $2.99 \pm 1.09^{* a}$ & $3.08 \pm 0.96^{¥ \mathrm{a}}$ & $1.37 \pm 0.36^{* 5}$ & 100 & - & 95 & 5 & - & 100 \\
\hline
\end{tabular}

Different symbols mean statistically significant differences between composite and giomer in each time interval (t-test, $P<0.05)$.

Different letters mean statistically significant differences between time intervals in each material (Repeated measurement ANOVA, $P<0.05)$. 
In addition, comparison of the shear bond strength of composite resin and MTA at each time interval with the use of t-test showed that:

-Immediately after mixing, there was no significant difference in shear bond strength between composite resin and giomer $(P=0.57)$.

-At 2.45-hour and 3 days after mixing, the shear bond strength of composite resin was significantly higher than that of giomer $(P=0.001)$ (Figs. 1,2).

\section{Discussion}

Literature on the direct pulp capping procedures indicates that one of the most important factors in the prognosis of pulp-capped teeth is to place a microleakage-free restoration immediately after the procedure. Therefore, the bond strength between the restorative material and the cavity liner is one of the most important factors in the quality of tooth restorations and the response of the pulp to the pulp capping procedure (16). Absence of a proper bond with a hermetic seal results in microleakage and penetration of bacteria into the pulp, giving rise to the failure of vital pulp therapy $(9,10)$.

In adhesive dentistry, microtensile shear bond strength ( $\mu$ TBS) test has been recommended for the evaluation of the bond strength. However, since MTA is a brittle material it is not possible to carry out $\mu$ TBS on it. Therefore, in the present study shear bond strength test, which has been used in the majority of previous studies, was used (11).

Since the initial setting time of MTA is 2 hours and 45

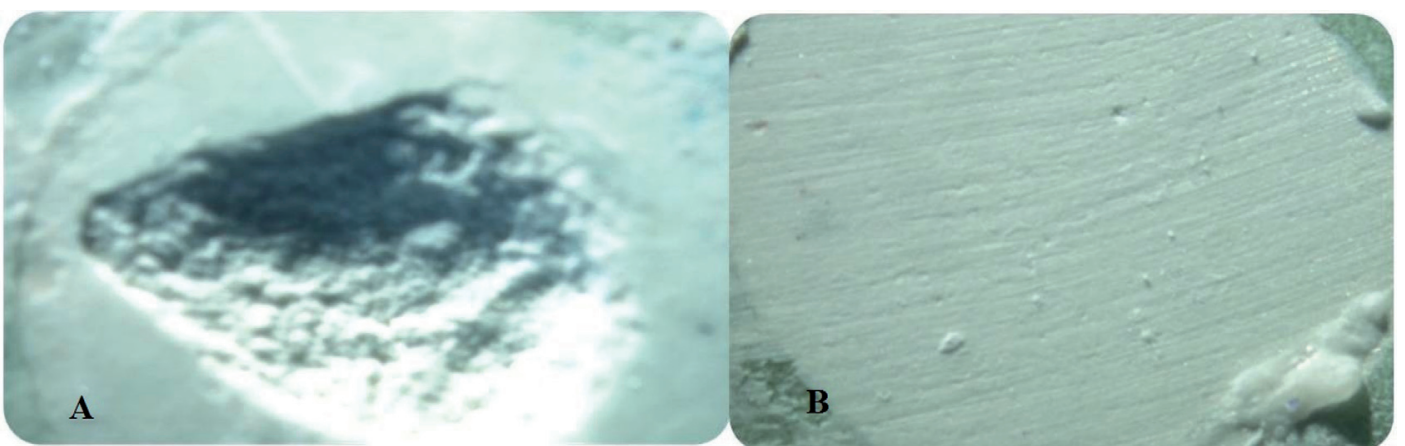

Fig. 1: Failure modes of interfaces between MTA with composite resin and giomer; A: Cohesive, B: Adhesive.

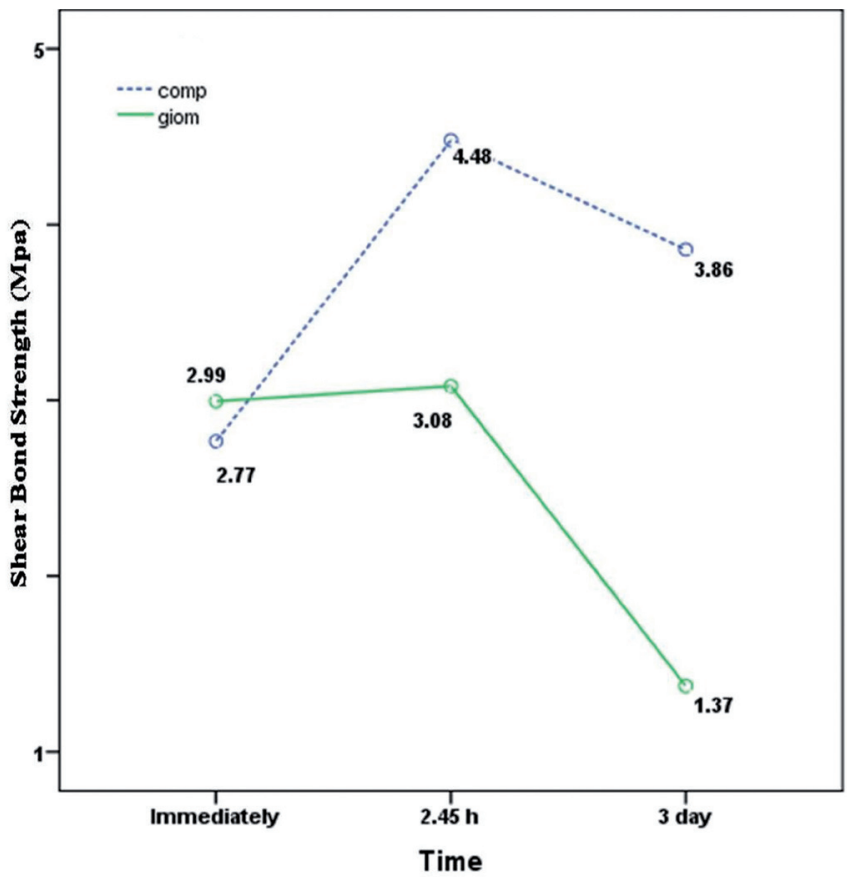

Fig. 2: Comparison of the shear bond strengths of giomer and composite resin at different time intervals. 
minutes and its final setting time is approximately 3 days (17), one basic question for any clinician is whether or not it is possible to avoid a 2 -session treatment, which is considered a risk factor for the success of vital pulp therapy, by placing composite resin or another adhesive material on the MTA immediately after mixing and placing it in the pulp capping area.

The results of the present study showed that the shear bond strength of composite resin (with E\&R adhesive system) to MTA was low immediately after mixing; however, it significantly increased 2.45 hours after mixing. In addition, the bond strength to completely set MTA (3 days) was not significantly different from that after the initial setting ( 2.45 hours).

On the other hand, the bond strength of giomer (one-step self-etch adhesive system) immediately and 2 hours and 45 minutes after mixing was similar and higher than that 3 days after mixing.

SEM studies have shown that etching the surface of WMTA with phosphoric acid results in the erosion of crystalline structures and creation of a cracked surface with internal cracks $(18,19)$. In contrast, use of selfetch systems does not result in the formation of cracks or pores on the MTA surface, although it destroys the crystalline structure. Therefore, it appears greater surface roughness is the reason for higher bond strength of composite resin to MTA with the use of ER adhesive systems compared to the use of two-step self-etch adhesive systems. Similar results have been reported in several previous studies $(12,20)$.

The decrease in bond strength over time in the giomer group might be attributed to the inability of the self-etch adhesive system to penetrate into the completely set MTA because in this system no separate step exists for etching with phosphoric acid and its mechanism of action depends on simultaneous etching, priming and bonding. In contrast, in the present study, the bond strength of composite resin increased over time, which might be attributed to the greater stability of the interfacial layer resulting from the penetration of the adhesive into the set MTA compared to the interfacial layer, resulting from the penetration of the adhesive into MTA immediately after mixing.

Neelakantan et al. showed that the bond strength of composite resin to MTA with the use of E\&R and one-step and two-step self-etch adhesive systems 24 hours after mixing decreased significantly compared to that immediately and 45 minutes after mixing (11). However, in the present study, the bond strengths of composite resin with the use of E\&R adhesive system and giomer with the use of one-step self-etch adhesive system immediately after mixing were not different significantly. Nonetheless, at 2-hour-and-45-minute and 3-day intervals the bond strength of composite resin was significantly higher than that of giomer.
Oskoee et al. showed that the type of the adhesive system) E\&R vs. 2-step self-etch) had no effect on the bond strength of composite resin to MTA after complete setting (10). In contrast, in a study by Shin et al., the highest bond strength of composite resin to MTA was achieved with the use of Adhes E One F one-step self-etch adhesive system; however, there was no significant difference between the system above and 3-step and one-bottle E\&R systems, with significantly higher bond strength compared to two-step self-etch adhesive systems (18). Neelakantan, too, reported a higher bond strength of composite resin to MTA with the use of one-step selfetch adhesive systems, which was attributed to factors such as $\mathrm{pH}$ or better wetting ability due to the simultaneous presence of solvents such as water and alcohol, resulting in a decrease in contact angle (11). The $\mathrm{pH}$ of the giomer system is 2.4. In addition, it lacks HEMA and acetone has replaced water and alcohol in its structure. Ethanol-based adhesive systems have higher bond strength compared to acetone-based adhesives ( $\mathrm{R}$ and the internal angles). In addition, some of the properties of acetone, such as high volatility and rapid evaporation when the bottle's cap is removed, make it an inappropriate solvent (21). In the self-etch adhesive systems, usually ethanol and acetone are used in combination with water as a solvent. A combination of ethanol or acetone with water is referred to as azeotropic, a property that forms hydrogen bonds between ethanol or acetone and water, facilitating the evaporation of this composition $(22,23)$; however, the capacity of the formation of hydrogen bonds with the ketone $(\mathrm{C}=\mathrm{O})$ in acetone is less than that of ethanol (OH-) (24).

Another interesting finding of the present study was the fact that the bond strengths of giomer and composite resin to MTA immediately after mixing were not significantly different from each other, consistent with the similar fracture patterns of MTA in the two groups $(100 \%$ cohesive). In contrast, at 2.45-hour and 3-day intervals, the bond strength of composite resin to giomer was significantly higher than that of giomer, as confirmed by fracture patterns because the higher number of adhesive failures indicates a weak bond strength. Adhesive failures in 2.45-hour and 3-day giomer groups increased in number, with the least resin bond strength in the 3-day giomer group between the study groups, and all the fractures were adhesive. In addition, the distribution pattern of fractures was highly consistent with the giomer bond strength; in this context, the $100 \%$ cohesive pattern in the immediate group converted to $100 \%$ adhesive fracture in the 3-day group.

Restorations subsequent to pulp capping procedures have different interfaces due to the multiplicity of the materials used, and the efficacy of the seal of each interface is important in the final success of the treatment. The bond strength values of different adhesive systems 
have been reported to be 13-35 MPa and the recommended bond strength values to achieve a restoration with no gaps with a proper seal has been reported to be 17-20 $\mathrm{MPa}(25,26)$, which is much higher than bond strength values of giomer and composite resin with MTA in the present study. On the other hand, several studies have reported that the bond strength of conventional glassionomer (GIC) to MTA is high for two reasons: Firstly, the surface of MTA is rich in metallic oxides with which the GIC can form chemical bonds through strong metallic bonds; secondly, porosities on the MTA surface increase the surface area of micromechanical interlocking between MTA and GIC (27-29).

However, studies have shown this interesting finding that if GIC is bonded to the MTA surface after mixing MTA, it will undergo pre-test failures (11) and it has been recommended that if a decision is made to cover the surface of MTA with GIC, this should be done at least 45 minutes after mixing and placement of MTA in the pulp cap area (20). Oskoee et al. reported that when MTA are used as pulp capping agents, it is advisable to cover them with RMGI before restoration with composite resin because the bond strength of RMGI to composite resin is significantly higher than that to MTA and CEM (9).

The chief limitation of the present study was a lack of evaluation of the characteristics and surface morphology of WMTA at different time intervals, which might have helped explain the results. Therefore, it is suggested that in future studies SEM and AFM evaluation be carried out in order to further elucidate the reasons for decreases and increases in bond strength at different time intervals.

\section{References}

1. Aguilar P, Linsuwanont P. Vital pulp therapy in vital permanent teeth with cariously exposed pulp: a systematic review. J Endod. 2011;37:581-7.

2. Trope M. Regenerative potential of dental pulp. Pediatr Dent. 2008;30:206-10.

3. Tziafas D, Pantelidou O, Alvanou A, Belibasakis G, Papadimitriou $\mathrm{S}$. The dentinogenic effect of mineral trioxide aggregate (MTA) in short-term capping experiments. Int Endod J. 2002;35:245-54.

4. Asgary S, Eghbal MJ. Treatment outcomes of pulpotomy in permanent molars with irreversible pulpitis using biomaterials: a multi-center randomized controlled trial. Acta Odontol Scand. 2013;71:130-6.

5. Eghbal MJ, Asgary S, Baglue RA, Parirokh M, Ghoddusi J. MTA pulpotomy of human permanent molars with irreversible pulpitis. Aust Endod J. 2009;35:4-8.

6. Min KS, Park HJ, Lee SK, Park SH, Hong CU, Kim HW, et al. Effect of mineral trioxide aggregate on dentin bridge formation and expression of dentin sialoprotein and heme oxygenase-1 in human dental pulp. J Endod. 2008;34:666-70.

7. Accorinte Mde L, Holland R, Reis A, Bortoluzzi MC, Murata SS, Dezan E Jr, et al. Evaluation of mineral trioxide aggregate and calcium hydroxide cement as pulp-capping agents in human teeth. J Endod. 2008;34:1-6.

8. Parolia A, Kundabala M, Rao NN, Acharya SR, Agrawal P, Mohan $\mathrm{M}$, et al. A comparative histological analysis of human pulp following direct pulp capping with Propolis, mineral trioxide aggregate and Dycal. Aust Dent J. 2010;55:59-64.
9. Oskoee SS, Kimyai S, Bahari M, Motahari P, Eghbal MJ, Asgary S. Comparison of shear bond strength of calcium-enriched mixture cement and mineral trioxide aggregate to composite resin. J Contemp Dent Pract. 2011;12:457-62.

10. Savadi Oskoee S, Bahari M, Kimyai S, Motahhari P, Eghbal MJ, Asgary S. Shear bond strength of calcium enriched mixture cement and mineral trioxide aggregate to composite resin with two different adhesive systems. J Dent (Tehran). 2014;11:665-71.

11. Neelakantan P, Grotra D, Subbarao CV, Garcia-Godoy F. The shear bond strength of resin-based composite to white mineral trioxide aggregate. J Am Dent Assoc. 2012;143:e40-5.

12. Tunc ES, Sonmez IS, Bayrak S, Egilmez T. The evaluation of bond strength of a composite and a compomer to white mineral trioxide aggregate with two different bonding systems. J Endod. 2008;34:603-5. 13. Gordan VV, Mondragon E, Watson RE, Garvan C, Mjor IA. A clinical evaluation of a self-etching primer and a giomer restorative material: results at eight years. J Am Dent Assoc. 2007;138:621-7.

14. Sunico MC, Shinkai K, Katoh Y. Two-year clinical performance of occlusal and cervical giomer restorations. Oper Dent. 2005;30:282-9.

15. Wilson NH, Gordan VV, Brunton PA, Wilson MA, Crisp RJ, Mjor IA. Two-centre evaluation of a resin composite/ self-etching restorative system: three-year findings. J Adhes Dent. 2006;8:47-51.

16. Cantekin K, Avci S. Evaluation of shear bond strength of two resinbased composites and glass ionomer cement to pure tricalcium silicate-based cement (Biodentine(R)). J Appl Oral Sci. 2014;22:302-6.

17. Parirokh M, Torabinejad M. Mineral trioxide aggregate: a comprehensive literature review--Part I: chemical, physical, and antibacterial properties. J Endod. 2010;36:16-27.

18. Shin JH, Jang JH, Park SH, Kim E. Effect of mineral trioxide aggregate surface treatments on morphology and bond strength to composite resin. J Endod. 2014;40:1210-6.

19. Kayahan MB, Nekoofar MH, Kazandag M, Canpolat C, Malkondu O, Kaptan F, et al. Effect of acid-etching procedure on selected physical properties of mineral trioxide aggregate. Int Endod J. 2009;42:1004-14.

20. Bayrak S, Tunc ES, Saroglu I, Egilmez T. Shear bond strengths of different adhesive systems to white mineral trioxide aggregate. Dent Mater J. 2009;28:62-7.

21. Cardoso Pde C, Lopes GC, Vieira LC, Baratieri LN. Effect of solvent type on microtensile bond strength of a total-etch one-bottle adhesive system to moist or dry dentin. Oper Dent. 2005;30:376-81.

22. Pashley EL, Zhang Y, Lockwood PE, Rueggeberg FA, Pashley DH. Effects of HEMA on water evaporation from water-HEMA mixtures. Dent Mater. 1998;14:6-10.

23. Imazato S, Russell RR, McCabe JF. Antibacterial activity of MDPB polymer incorporated in dental resin. J Dent. 1995;23:177-81.

24. Van Landuyt KL, Snauwaert J, De Munck J, Peumans M, Yoshida Y, Poitevin A, et al. Systematic review of the chemical composition of contemporary dental adhesives. Biomaterials. 2007;28:3757-85.

25. Davidson CL, de Gee AJ, Feilzer A. The competition between the composite-dentin bond strength and the polymerization contraction stress. J Dent Res. 1984;63:1396-9.

26. Teixeira CS, Chain MC. Evaluation of shear bond strength between self-etching adhesive systems and dentin and analysis of the resin-dentin interface. Gen Dent. 2010;58:e52-61.

27. Ballal S, Venkateshbabu N, Nandini S, Kandaswamy D. An in vitro study to assess the setting and surface crazing of conventional glass ionomer cement when layered over partially set mineral trioxide aggregate. J Endod. 2008;34:478-80.

28. Nandini S, Ballal S, Kandaswamy D. Influence of glass-ionomer cement on the interface and setting reaction of mineral trioxide aggregate when used as a furcal repair material using laser Raman spectroscopic analysis. J Endod. 2007;33:167-72.

29. Camilleri J, Pitt Ford TR. Mineral trioxide aggregate: a review of the constituents and biological properties of the material. Int Endod J. 2006;39:747-54.

\section{Conflict of Interest}

The authors have declared that no conflict of interest exist. 